\title{
A wind-tunnel study of flow distortion at a meteorological sensor on top of the BT Tower, London, UK
}

Article

Accepted Version

Barlow, J. F., Harrison, J., Robins, A. G. and Wood, C. R. (2011) A wind-tunnel study of flow distortion at a meteorological sensor on top of the BT Tower, London, UK. Journal of Wind Engineering and Industrial Aerodynamics, 99 (9). pp. 899-907. ISSN 0167-6105 doi:

https://doi.org/10.1016/j.jweia.2011.05.001 Available at https://centaur.reading.ac.uk/22779/

It is advisable to refer to the publisher's version if you intend to cite from the work. See Guidance on citing.

Published version at: http://dx.doi.org/10.1016/j.jweia.2011.05.001

To link to this article DOI: http://dx.doi.org/10.1016/j.jweia.2011.05.001

Publisher: Elsevier

Publisher statement: 9th UK Conference on Wind Engineering (September, 2010)

All outputs in CentAUR are protected by Intellectual Property Rights law, including copyright law. Copyright and IPR is retained by the creators or other copyright holders. Terms and conditions for use of this material are defined in the End User Agreement. 


\section{CentAUR}

Central Archive at the University of Reading

Reading's research outputs online 


\title{
A wind-tunnel study of flow distortion at a meteorological sensor on top of the BT Tower, London, UK
}

\author{
Janet F. Barlow ${ }^{\mathrm{a}}$, James Harrison ${ }^{\mathrm{b}}$, Alan G. Robins ${ }^{\mathrm{b}}$, and Curtis R. Wood ${ }^{\mathrm{a}}$ \\ ${ }^{\mathrm{a}}$ Department of Meteorology, University of Reading, Earley Gate, PO Box 243, \\ Reading RG6 6BB, UK (j.f.barlow@reading.ac.uk; c.r.wood@reading.ac.uk) \\ ${ }^{\mathrm{b}}$ School of Engineering, University of Surrey, Guildford GU2 7XH, UK \\ (a.robins@surrey.ac.uk ) \\ * corresponding author: telephone $+44(0) 118$ 3786022; fax $+44(0) 1183788905$
}

\begin{abstract}
High quality wind measurements in cities are needed for numerous applications including wind engineering. Such data-sets are rare and measurement platforms may not be optimal for meteorological observations. Two years' wind data were collected on the BT Tower, London, UK, showing upward deflection for all wind directions. Wind tunnel simulations were performed to investigate flow distortion around two scale models of the Tower. Using a 1:160 scale model it was shown that the Tower causes a small deflection (c. $0.5^{\circ}$ ) compared to the lattice on top on which the instruments were placed (c. 0 to $4^{\circ}$ ). These deflections may have been underestimated due to wind tunnel blockage. Using a 1:40 model, the observed flow pattern was consistent with streamwise vortex pairs shed from the upstream lattice edge.

Correction factors were derived for different wind directions and reduced deflection in the full-scale data-set by $<3^{\circ}$. Instrumental tilt caused a sinusoidal variation in deflection of c. $2^{\circ}$. The residual deflection $\left(\mathrm{c} .3^{\circ}\right)$ was attributed to the Tower itself. Correction of the wind-speeds was small (average 1\%) therefore it was deduced that flow distortion does not significantly affect the measured wind-speeds and the wind climate statistics are reliable.
\end{abstract}




\section{Introduction}

Knowledge of the wind climate in urban areas is required by wind engineers for many applications, including the design of new buildings and infrastructure. However, there are few reliable data-sets available, in part due to the traditional view of national meteorological services that wind measurements in urban areas are invalidated by the microclimatic effects of the buildings. With growing interest in urban climatology in its own right, new guidelines have emerged in terms of siting meteorological stations with the aim of making representative meteorological measurements in urban areas (Oke, 2008).

The response of the wind to an urban surface is complex, depending on surface roughness, stability, the horizontal extent of the urban area, etc. (Roth, 2000). Measurements near ground level are limited in their representativeness, being influenced by immediate obstacles or local urban characteristics. This layer, called the roughness sub-layer, extends up to 2 to 5 times the local mean building height, $H$, (Rotach 1999; Barlow and Coceal 2009). Above this height, meteorological measurements are influenced by the area-averaged roughness of the surface in a given "neighbourhood", within an area of several square kilometres. Such high level measurements are likely to be more representative of urban wind climate than nearby airports, the data from which are often described as "urban" despite the airports being sometimes tens of kilometres from the city centre. One example is the London Heathrow airport meteorological station which is often used to represent "London's" climate.

Data-sets are emerging from urban measurement platforms for longer periods than the few weeks of a field campaign. The BUBBLE campaign in Basel (Christen, 2005) saw the use of several instrumentation towers with heights up to $2.5 \mathrm{H}$ over a period of one year. Roth (2000) reviewed turbulence measurements largely between 0.7 and $6.3 \mathrm{H}$. However, measurements on much higher platforms - usually telecommunications towers or large buildings - have also been used (e.g. Al-Jiboori et al. 2002, up to $280 \mathrm{~m}$ over Beijing). Wood et al. (2010) published turbulence data for the BT Tower in central London, at a height of $190 \mathrm{~m}$. Barlow et al. (2009) compared roof-top measured data with BT Tower data in a discussion of how to reference such localized wind-speed measurements to the better exposed reference above the city. 
The value of such high level platforms is clear, especially if they can be maintained and run for longer periods. However, the quality of the data can depend on discerning any flow distortion due to the structure on which they are mounted. Griessbaum and Schmidt (2009) proposed an LES-based methodology to correct data obtained on a tower at $65 \mathrm{~m}$ in Münster, Germany. This paper presents an investigation based on wind tunnel simulations into flow distortion around the instruments used by Wood et al. (2010) on the BT Tower. Correction factors are derived, their significance is discussed and the resulting revised wind climatology is presented.

\section{Description of site and full-scale data}

\subsection{BT Tower site and equipment}

A full description of the BT Tower site and turbulence data collected between 2006 and 2008 is given in Wood et al. (2010), but the key points are given here.

A sonic anemometer was mounted at $190 \mathrm{~m}$ on the BT telecommunications tower in west-central London $\left(51.5215^{\circ} \mathrm{N}, 0.1389^{\circ} \mathrm{W}\right)$. The BT Tower is the tallest building within several kilometres of its dense urban surroundings (and currently the fourth tallest in London), with good exposure to winds in all directions. Wood et al. (2010) estimated the source area (Schmid, 1997) for measurements at the Tower and found it to lie between 1 and $10 \mathrm{~km}$ radius, for neutral stability conditions. Within $1-10 \mathrm{~km}$ of the Tower the mean building height is $8.8 \pm 3.0 \mathrm{~m}$; beyond $10 \mathrm{~km}$, mean height in suburban Greater London is $5.6 \pm 1.8 \mathrm{~m}$. Hence, the sensor is $\sim 22$ times the mean building height upwind of the tower between 1 and10 km radius. Data were collected from October 2006 to May 2008. The three-component winds $(u, v, w)$ and sonic temperature $\left(T_{s}\right)$ from the sonic anemometer (R3-50, Gill Instruments, $0.01 \mathrm{~m} \mathrm{~s}^{-1}$ resolution and accuracy) were archived at $20 \mathrm{~Hz}$. For this study, data were averaged over 30 minute periods. It should be noted that the wind-speed is computed as $U=\sqrt{\left(\bar{u}^{2}+\bar{v}^{2}+\bar{w}^{2}\right)}$, that is to say the vertical component is included in the calculation: a cup anemometer would only measure the purely horizontal component of the wind vector. 
Fig. 1a shows the BT Tower. The anemometer was mounted on a vertical pole attached to the top of an open lattice scaffolding tower of $12.3 \mathrm{~m}$ height on top of the main structure (see Fig.1b showing southern aspect). The Tower is circular in crosssection: the diameter of the very top section is $12 \mathrm{~m}$, and the maximum diameter is $19.75 \mathrm{~m}$. Fig. 1c shows a plan view of the lattice tower and indicates its orientation with respect to north. The location of the anemometer is indicated.

\subsection{Evidence of flow distortion from full-scale data}

Fig. 2 shows the wind-rose and frequency distribution of wind-speeds as measured at the BT Tower. It can be seen that the dominant wind direction is south westerly, with $27 \%$ of the data-set (out of a total of 1841230 minute periods) lying in this sector. The strongest wind-speeds are associated with this sector, with an average of $9.5 \mathrm{~ms}^{-1}$. The frequency distribution of wind-speed (Fig. 2b) has a mean wind-speed of $8.05 \mathrm{~m}$ $\mathrm{s}^{-1}$, median of $7.85 \mathrm{~m} \mathrm{~s}^{-1}$ and the $90 \%$ percentile wind-speed is $13.08 \mathrm{~m} \mathrm{~s}^{-1}$. A Weibull distribution fits the data well, with shape factor $\mathrm{k}=2.24$ and a scale factor of $9.08 \mathrm{~m}$ $\mathrm{s}^{-1}$.

For each 30 minute period a vertical deflection angle was defined as $\psi=$ $\sin ^{-1}(w / U)$ where $w$ is the vertical component and $U$ is the magnitude of the wind vector. Any deviation away from zero indicates a local perturbation to the flow, either due to flow distortion around the Tower, or a tilt of the instrument away from vertical. Fig. 3a shows the vertical deflection angle as a function of wind-speed. It can be seen that there is wide scatter at low wind-speeds, when buoyant effects may be acting, causing large up- and down- draughts. As wind-speed increases, scatter decreases as conditions tend to near-neutral, and the average vertical deflection angle lies between 6 and $8^{\circ}$. Fig. $3 \mathrm{~b}$ shows $\psi$ as a function of wind bearing, with data highlighted for wind-speeds above the median value, $U>7.85 \mathrm{~ms}^{-1}$. It can clearly be seen that the flow is positively inclined for all angles, and there is a sinusoidal tendency. Whether the flow is merely tilted or is indeed being accelerated requires specific modelling of the flow distortion due to the Tower itself, before any corrections can be considered.

\section{Wind tunnel modelling}




\subsection{Wind tunnel and measurement methods}

Wind tunnel modelling took place in the "A" tunnel of the Environmental Flow Research Laboratory (EnFlo) at the University of Surrey, UK. The tunnel is an open circuit blow-down tunnel and has dimensions $602 \times 897 \times 5000 \mathrm{~mm}($ depth $\times$ width $\times$ length), a maximum operating velocity of $15 \mathrm{~ms}^{-1}$ and free-stream turbulence of less than $0.1 \%$. The co-ordinate system used is Cartesian with $x$ axis along the tunnel length and parallel to the flow with zero located at the centre of each model, $y$ axis lateral to the flow and $z$ vertically perpendicular to the flow (see Fig. 1c). Each model was placed on the wind tunnel centreline at a distance of $1.3 \mathrm{~m}$ downstream of the start of the working section. Instrumentation used was a Pitot-static tube, located upstream of the models that monitored free-stream wind-speed which was held at 10 $\mathrm{m} \mathrm{s}^{-1} \pm 2 \%$ by feedback control on the tunnel fan. Upstream of the models, a uniform velocity field was used.

Single hot wire (Dantec P11) and cross-wire (Dantec P61) anemometers were both used for detailed mapping of the flow around the models, run using a University of Newcastle (Australia) bridge unit. A micro-manometer (Furness, FC012) was used with a standard Pitot-static tube to provide reference wind-speed measurements. The hot wire anemometers were automatically calibrated against the Pitot-static tube every hour if measurement runs exceeded this duration. Data were logged using a bespoke program written using LabView software.

\subsection{Models of BT Tower}

Flow around the BT Tower was investigated at two scales: 1:160, modelling the top third of the Tower to assess the bulk impact of the Tower on the flow; and 1:40 scale, modelling the $12 \mathrm{~m}$ high lattice tower on which the equipment was mounted to determine any effect it might have on the wind-field. Fig. 4 shows photographs of the two models.

\section{$3.21: 160$ scale model}


Only the top third of the BT Tower itself was modelled at a scale of 1:160. The Tower section of the model was $233 \mathrm{~mm}$ high and machined from aluminium. The shape was simplified to capture only the main features and had a maximum diameter of 141.2 $\mathrm{mm}$. The lattice tower at the top was made of $1.75 \mathrm{~mm}$ card and galvanised steel wire. It was $82 \mathrm{~mm}$ in height, giving an overall model height of $315 \mathrm{~mm}$. The area blockage ratio of this model in the wind tunnel was $4.8 \%$ which was large enough to require an assessment of any local acceleration of the flow that might have resulted.

An experiment was first conducted to measure the wind profile above three cylinders with dimensions as shown in Table 1, the largest being of similar size to the 1:160 model. Flows of 6 and $12 \mathrm{~m} \mathrm{~s}^{-1}$ were used to test Reynolds number independence and the resulting profiles are shown in Fig. 5a. The profiles have been normalised using the local free-stream wind-speed, as it was observed that the flow was accelerated above the largest model. It can be seen that there is a good collapse of the data when scaled using local free-stream wind-speed, showing little distortion of the expected flow profile above a cylinder due to blockage, and that the influence of the cylinder on the flow above mostly occurs within one diameter $D$. In terms of a specific correction to be applied to the 1:160 model data, it was found that the local freestream velocity $U_{\text {fsloc }}=1.05 U_{\text {ref. }}$

Fig. 5b shows the vertical deflection angle as a function of normalised height for the cylinders. In this case it can be seen that the magnitude of the deflection is reduced in the case of the large cylinder, particularly for $(z-h)>0.25 D$. This is probably due to the blockage effect, indicating that deflection angles measured above the 1:160 model may also be affected. Flow measurements were made with and without the lattice tower on the 1:160 model. Fig 6 shows the vertical deflection angle as a function of height above the model in the two cases. For the Tower alone the pattern is similar to the case of the cylinders with small differences in magnitude. At the equivalent height of the instruments $(320 \mathrm{~mm})$ it can be seen that there is a small upward deflection due to the Tower $\left(\sim 0.5^{\circ}\right)$. When the lattice is added, the pattern is accentuated, showing a region of very large shear within $5 \mathrm{~mm}$ of the top of the lattice ( $315 \mathrm{up}$ to $320 \mathrm{~mm}$ ) where the vertical deflection due to both Tower and lattice tower is between 0 and $4^{\circ}$. As the sonic head is mounted at $0.6 \mathrm{~m}$ above the top of the lattice tower at full-scale 
(equivalent to approximately $5 \mathrm{~mm}$ at $1: 160$ scale) this is a region which must be modelled accurately, hence the need for a larger scale model.

Table 1also shows that in all cases $\operatorname{Re}<2 \times 10^{5}$ which is the upper threshold value for the sub-critical flow regime for flow around a cylinder. Thus it is likely that the wake generated by the 1:160 model is considerably wider laterally than for the BT Tower itself, for which $\operatorname{Re} \sim 1 \times 10^{7}$. Vortex shedding is unlikely to be a coherent feature of flow around the 1:160 model as the top part of the model is complex and likely instead to lead to a turbulent wake. For the purposes of this study, it is the flow around the very top where instruments were located which should be most faithfully reproduced

It is clear that the lattice tower itself has a significant effect on the flow whilst the effect of the main tower itself is relatively small at the height of the measurements. The next section describes the experiments focused around the 1:40 scale model of the lattice tower alone.

\section{$3.31: 40$ model}

The 1:40 scale model of the lattice tower was produced using rapid prototyping and was constructed from hard plastic. The struts in the lattice were circular in crosssection to mimic the scaffolding used at full-scale and had diameters of either 2 or 4 $\mathrm{mm}$. In the wind tunnel this gave $\operatorname{Re} \sim 1$ to $3 \times 10^{3}$, equivalent to 5 to $10 \times 10^{4}$ at fullscale. Both model and the full-scale Re indicate that the flow is subcritical with regard to the wake shed by each lattice strut and indicate that there is no need to provide extra roughness on the 1:40 wind tunnel model. The lattice is also porous enough that the wakes of individual struts are important in perturbing the flow near to where instruments are likely to be located, and the overall wake from the lattice is of less interest. Blockage effects were also deemed negligible for this model.

Fig. 7 shows the vertical deflection angle around the 1:40 model for an incident wind angle of $0^{\circ}$ for a) the $x-z$ plane at various heights and b) the $y-z$ plane at a height of 5 $\mathrm{mm}$ above the lattice. Note that the lattice is square in section with each side of length 
$72 \mathrm{~mm}$ at the top. It can seen from Fig 7a that there is a strong up-draught just above the leading edge and smaller up-draught at the downstream edge of the lattice. These perturbations decay quite quickly with height, but persist some distance downstream. The sonic anemometer height is equivalent to $16 \mathrm{~mm}$ above the lattice at this scale, so the maximum upward deflection experienced at that height lies between 4 and $7^{\circ}$.

Fig $7 \mathrm{~b}$ shows the vertical deflection angle for the $y$-z plane taken at different $x$ positions with respect to the lattice (where $x=0$ is the centre of the lattice). It can be seen that there is a complex series of up- and down-draughts which line up with the edges of the lattice tower at $y= \pm 35 \mathrm{~mm}$. At the leading edge $(x=-35 \mathrm{~mm})$, the maximum up-draught is seen and is relatively uniform across the width of the lattice. At points downstream of this, distinct up- and down-draughts develop, most likely as a consequence of streamwise vortex pairs generated at the upstream corners of the lattice. Lateral deflections were observed consistent with this hypothesis but are not presented here.

\section{Flow corrections}

\subsection{Method}

The 1:40 model data were used to devise flow corrections to account for distortion due to the lattice tower alone. The position of the sonic as used during Wood et al. 2010 was simulated, i.e. a position of $(x, y, z)=(-43.5,-28.5,329)$ corresponding to the red cross shown in Fig. 1c. To allow for model or positioning errors, points around this simulated position were also measured and an average over a small volume taken to give a more robust velocity estimate. The volume size was on average $(5 \mathrm{~mm})^{3}$ representing $(0.2 \mathrm{~m})^{3}$ at full-scale.

The methodology used is based on that of Griessbaum and Schmidt (2009) who used LES to simulate flow distortion around a similar tower with a meteorological mast. The methodology as used here corrects only the mean flow vectors. Wind-speed measured at the location of the instrument on the model is given by $\boldsymbol{U}=(u, v, w)$ where components are aligned with the $x, y$ and $z$ axes respectively. The reference 
flow vector upstream $\boldsymbol{U}_{\text {ref }}=\left(u_{\text {ref }}, v_{\text {ref }}, w_{\text {ref }}\right)=(10,0,0)$ for the duration of the experiments. A correction vector was defined according to

$$
\boldsymbol{F}=\left(\begin{array}{c}
f_{u} \\
f_{v} \\
f_{w}
\end{array}\right)=|\boldsymbol{U}|^{-1}\left(\begin{array}{c}
u-u_{\text {ref }} \\
v-v_{\text {ref }} \\
w-w_{\text {ref }}
\end{array}\right)
$$

The corrected wind vector is thus given by

$\boldsymbol{U}_{c}=\boldsymbol{U}_{m}-\left|\boldsymbol{U}_{m}\right| \boldsymbol{F}$

where $\boldsymbol{U}_{\boldsymbol{m}}$ is the measured wind vector. The correction vector $\boldsymbol{F}$ was evaluated for incident wind direction angles at $45^{\circ}$ intervals starting from $0^{\circ}$ using a Cartesian coordinate system as defined in Fig. 1c. The components of $\boldsymbol{F}$ are shown in Fig. 8, effectively showing the magnitude of the flow distortion at the location of the sonic anemometer. It can be seen that the $u$ and $w$ component corrections are in anti-phase, being largest and smallest respectively for an incident flow of $180^{\circ}$ when the sonic anemometer is on the lee-side of the lattice tower. The factors show a consistent acceleration (for $u$ ) and up-draught (for $w$ ) for all incident wind angles. For $v$ the flow is less symmetrical, probably due to the sonic anemometer being offset to the right of the centreline of the lattice tower: flow deviates slightly to the left when the sonic is on the windward side, and slightly to the right for the leeward side.

\subsection{Application to full-scale data}

Fig. 9 shows the original BT Tower vertical deflection angle data for $U>7.85 \mathrm{~m} \mathrm{~s}^{-1}$ (as in Fig. 3b) and the corrected data after application of the correction vector $\boldsymbol{F}$. It can be seen that the lattice tower accounts for approximately $2-3^{\circ}$ of the up-draught but there is still a substantial residual flow distortion.

It can be seen that the data-points follow an approximately sinusoidal curve. A function of the form $\psi=A \sin (\phi-\alpha)+B$ has been fitted to the data, giving best fit values of $A=2.31^{\circ}, B=3.36^{\circ}$ and $\alpha=166^{\circ}$. If the sonic anemometer was mounted with 
a slight tilt from vertical, then such a form is expected in the vertical deflection angle data. This seems to be the case, and thus $A$ can be interpreted as the average tilt angle, and $\alpha-90^{\circ}$ is the direction towards which the sonic anemometer is tilting, i.e. towards the north-east. It is conceivable that the instrument was leaning slightly as access to the instrumentation was only given very occasionally to local riggers attaching equipment to the lattice tower: notwithstanding, this is a very small tilt angle. The offset $B$ could be interpreted as the up-draught due to the Tower itself, averaged over all wind directions. At $3.36^{\circ}$ it is a comparable contribution to the upward deflection of the wind vector by the lattice.

In terms of overall changes to the statistics of the distribution, Table 2 shows the parameters of the Weibull distribution fitted to the wind-speeds before and after correction. Given confidence intervals of $95 \%$, it can be seen that there is a significant difference in scale factor, but no significant difference in shape factor. The reduction in the strongest wind-speeds can be interpreted by the percentiles: the $90^{\text {th }}$ percentile of the uncorrected data is $13.08 \mathrm{~m} \mathrm{~s}^{-1}$, which reduces to $12.81 \mathrm{~m} \mathrm{~s}^{-1}$ after correction. The median wind-speed reduces from $7.85 \mathrm{~m} \mathrm{~s}^{-1}$ to $7.77 \mathrm{~m} \mathrm{~s}^{-1}$. It can be seen that applying the correction factors has made very small differences to the wind-speed magnitude: the mean correction across the whole data-set was $\sim 1 \%$.

\section{Conclusions}

There is a need for high quality wind climate measurements in cities for numerous applications including building design, air quality modelling and wind engineering. However, such data-sets are rare and measurement platforms may not be optimal for meteorological observations. Wind tunnel simulations were performed of the flow around two scale models of the BT Tower in London, UK. This was done to investigate the significance of the flow distortion evident in the full-scale data of Wood et al. (2010) measured from October 2006 to May 2008, and to attempt to derive correction factors. 
The flow distortion manifested itself most strongly in a non-zero upward vertical deflection for all incident wind directions. Using a 1:160 model of the upper third of the Tower, it was seen that the Tower induced a relatively small upward deflection $\left(0.5^{\circ}\right)$ at the equivalent height of the instruments. When the lattice was included, a strong shear was observed at the same height with an upward deflection between 0 and $4^{\circ}$. A larger scale model (1:40) of the lattice alone was then used to investigate the effects of the lattice in greater detail. Quasi-periodic deflections, both vertical and lateral, were seen in the flow just above the lattice. These were thought to be consistent with the presence of streamwise vortex pairs generated at the upstream corners of the lattice.

The incident wind angle was varied to cover all wind sectors. Correction factors for all three components of the wind vector were derived and applied to the original data. The correction accounted for approximately $2-3^{\circ}$ of the original flow deflection. There was a residual quasi-sinusoidal variation in angle: in theory this could be explained by a tilt in the sonic anemometer. By fitting a sine curve, the tilt was estimated to be $2.31^{\circ}$ towards the north-east.

The remaining constant offset of $3.36^{\circ}$ was attributed to the up-draught induced by the Tower itself. This is a relatively large deflection compared to the estimates from the 1:160 simulations (c. 0.5 degrees). Blockage tests with different sizes of cylinders suggested that the vertical deflection may be underestimated in the case of significant blockage $(>5 \%)$, therefore the deflection due to the 1:160 Tower model may well be underestimated too.

Assuming that the main flow acceleration was due to the lattice tower itself, the change in wind-speed statistics was relatively small. Only the scale factor in the Weibull distribution was significantly affected, and the $90^{\text {th }}$ percentile wind-speed was reduced by $2 \%$. Given these relatively small changes it can be deduced that the measurement platform gives reliable wind-speed data over the city despite a small amount of flow distortion. The wind tunnel simulations proved useful in explaining most of the behaviour of the full-scale flow data, and in computing useful quantitative corrections, despite certain limitations due to blockage. 


\section{Acknowledgements}

We wish to acknowledge the help of Paul Hayden in the execution of the experiments and Allan Wells in the design of the models. David Joyson also carried out the initial study using the 1:160 model as part of his undergraduate studies which then led onto the present study which was completed by James Harrison as part of an EPSRCfunded summer placement.

\section{References}

Al-Jiboori, M.H., Xu, Y.M., Qian, Y.F., 2002. Local similarity relationships in the urban boundary layer. Boundary-Layer Meteorology 102(1), 63-82

Barlow, J.F., Coceal, O., 2009. A review of urban roughness sublayer turbulence. UK Met Office Technical Report no. 527, 68 pp.

Barlow, J.F., Dobre, A., Smalley, R.J., Arnold, S.J., Tomlin, A.S., Belcher, S.E., 2009. Referencing of street-level flows measured during the DAPPLE 2004 campaign. Atmospheric Environment 43, 5536-5544.

Christen, A., 2005. Atmospheric turbulence and surface energy exchange in urban environments. PhD thesis, Faculty of Science, University of Basel, 142 pp.

Griessbaum, F., Schmidt, A., 2009. Advanced tilt correction from flow distortion effects on turbulent $\mathrm{CO} 2$ fluxes in complex environments using large eddy simulation. Quarterly Journal of the Royal Meteorological Society 135, 16031613.

Oke, T.R. 2008. Urban Observations, in: WMO No. 8, Guide to meteorological instruments and methods of observations, $7^{\text {th }}$ ed., World Meteorological Organization.

Rotach, M.W., 1999. On the influence of the urban roughness sublayer on turbulence and dispersion. Atmospheric Environment 33, 4001-4008.

Roth, M., 2000. Review of atmospheric turbulence over cities. Quarterly Journal of the Royal Meteorological Society 126, 941-990.

Schmid, H.P., 1997. Experimental design for flux measurements: matching scales of observation and fluxes. Agricultural and Forest Meteorology 87, 179-200. 
Wood, C.R., Lacser A., Barlow, J.F., Padhra, A., Belcher, S.E., Nemitz, E., Helfter, C., Famulari, D., Grimmond, C.S.B., 2010. Turbulent flow at 190 metres above London during 2006-2008: a climatology and the applicability of similarity theory. Boundary-Layer Meteorology 137, 77-96. 


\begin{tabular}{|c|c|c|c|c|c|}
\hline Cylinder & $\begin{array}{c}\text { Height } \\
(\mathbf{m m})\end{array}$ & $\begin{array}{c}\text { Diameter } \\
\mathbf{( m m})\end{array}$ & $\begin{array}{c}\text { Reynolds no. } \\
\times \mathbf{1 0}^{\mathbf{4}} \\
\left(\mathbf{6 / 1 2} \mathbf{~ m ~ s}^{-\mathbf{1}}\right)\end{array}$ & $\begin{array}{c}\text { Cross- } \\
\text { sectional } \\
\text { area }\left(\mathbf{m m}^{\mathbf{2}}\right)\end{array}$ & $\begin{array}{c}\text { Area } \\
\text { blockage } \\
\text { ratio (\%) }\end{array}$ \\
\hline Small & 118 & 60 & $2.4 / 4.8$ & 7080 & 1.3 \\
Medium & 234 & 89 & $3.5 / 7.1$ & 20800 & 3.9 \\
Large & 235 & 141 & $5.6 / 11.2$ & 33100 & 6.1 \\
1:160 model & 233 & $111(\mathrm{avg})$ & For $10 \mathrm{~m} \mathrm{~s}^{-1}: 7.4$ & 25900 & 4.8 \\
\hline
\end{tabular}

${ }^{a}$ viscosity calculated assuming temperature $\mathrm{T}=20^{\circ} \mathrm{C}$

Table 1: Dimensions of test cylinders used for blockage tests. Dimensions of 1:160 BT Tower model included for comparison. 


\begin{tabular}{|l|l|l|l|l|}
\hline data & $\boldsymbol{\lambda}$ (scale factor) & CI (low,high) & $\mathbf{k}$ (shape factor) & CI(low,high) \\
\hline uncorrected & 9.083 & $9.022,9.145$ & 2.240 & $2.214,2.265$ \\
\hline corrected & 8.956 & $8.896,9.016$ & 2.269 & $2.243,2.295$ \\
\hline
\end{tabular}

Table 2: Coefficients of Weibull distribution fitted to uncorrected and corrected windspeed data. CI: 95\% confidence interval. 


\section{Figure captions}

Figure 1: The BT Tower, London, UK a) photo of Tower b) photo of lattice tower at the top c) plan view of lattice showing orientation with respect to north. Also indicated are the co-ordinate system used in the wind tunnel and the location of the meteorological instrumentation.

Figure 2: Observed wind 2006 - 2008 a) frequency of wind direction by sector b) frequency of wind-speeds.

Figure 3: Observed vertical deflection angle a) as a function of wind-speed b) as a function of wind direction (black points indicate $U>7.85 \mathrm{~m} \mathrm{~s}^{-1}$ ).

Figure 4: Photographs of wind tunnel models a) 1:160 scale b) 1:40 scale.

Figure 5: Profiles above different-sized cylinders a) normalized wind-speed b) vertical deflection angle.

Figure 6: Vertical deflection angle measured above the 1:160 model, both with and without lattice.

Figure 7: Vertical deflection angle measured above the 1:40 model a) in the $x$-z plane at different heights along the centreline (where $z$ ' is the height above the lattice) b) in the $y-z$ plane at different $x$ positions for a height $5 \mathrm{~mm}$ above the lattice.

Figure 8: Flow correction factors for wind components $u, v$ and $w$ as a function of incident wind angle.

Figure 9: Observed deflection angles for $U>7.85 \mathrm{~m} \mathrm{~s}^{-1}$ with (grey) and without (black) correction factors applied. Fitted sinusoidal curve indicates instrumental tilt. 
Figure(s)

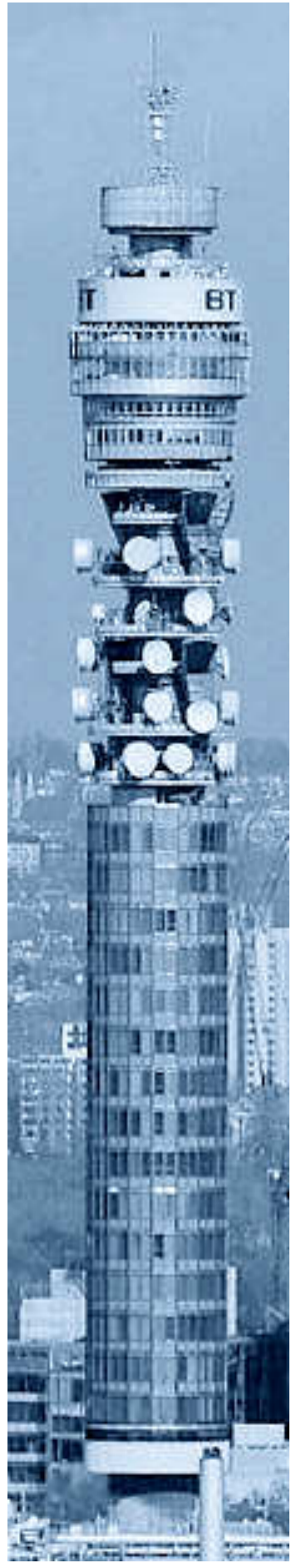




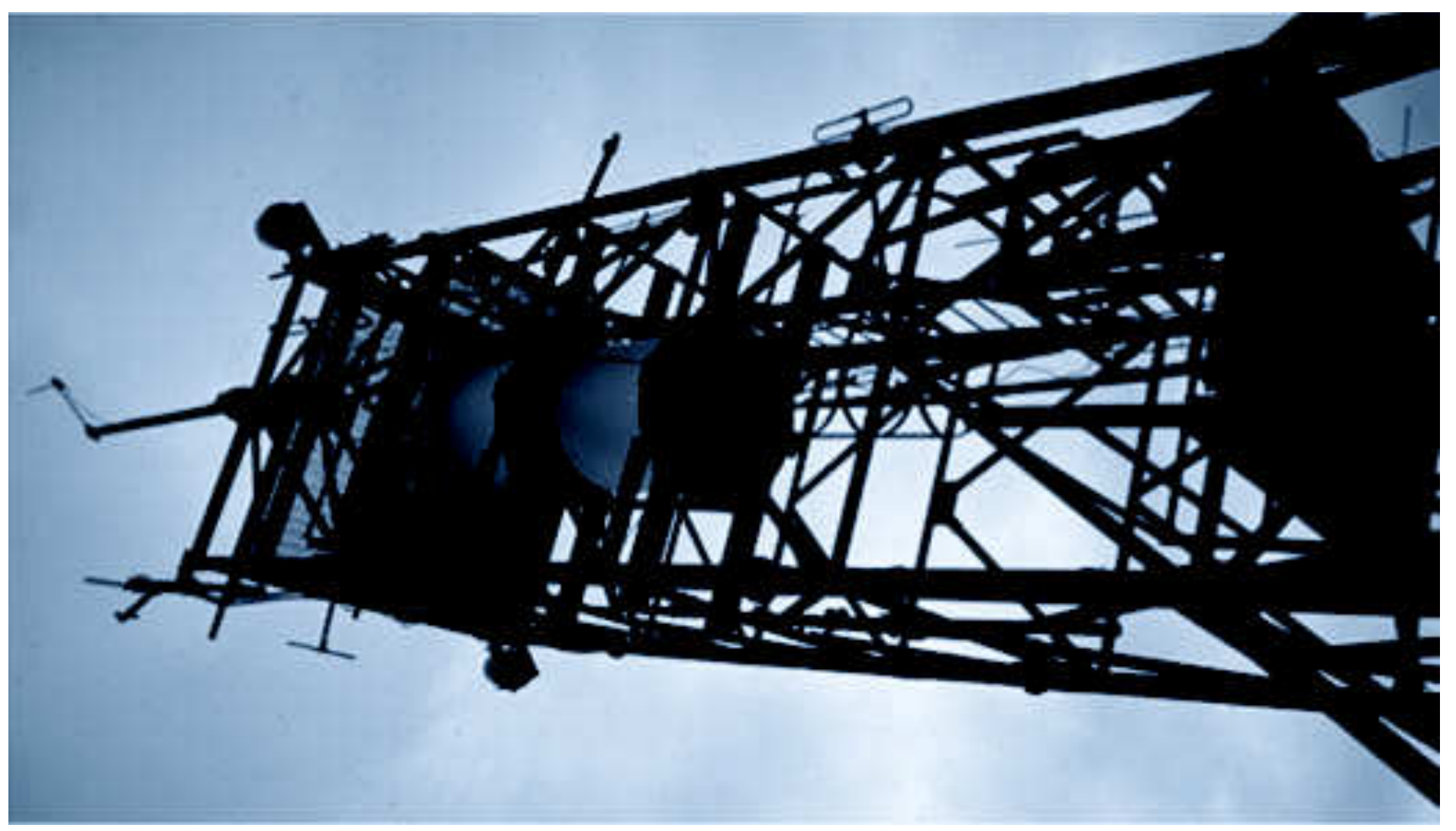




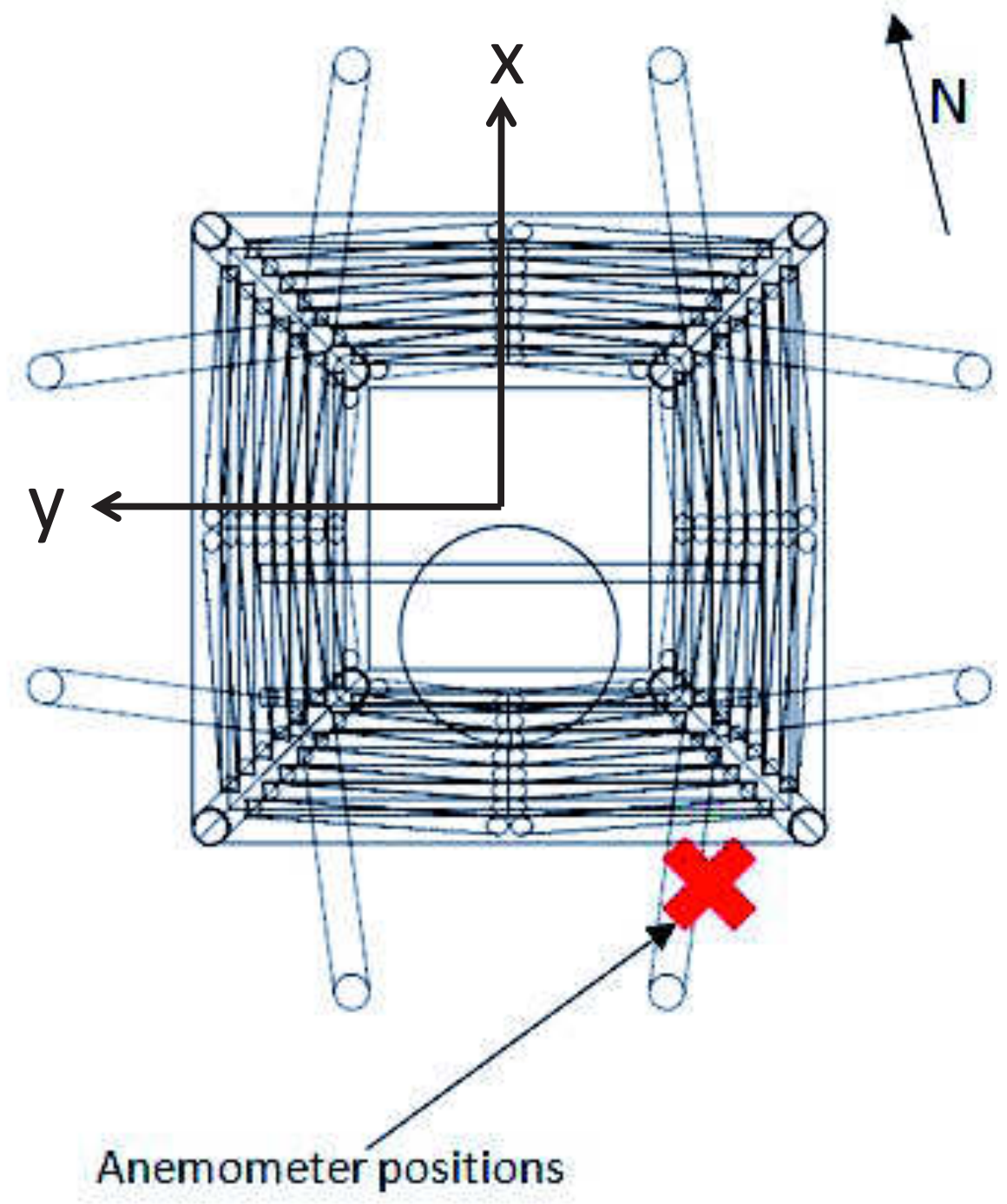




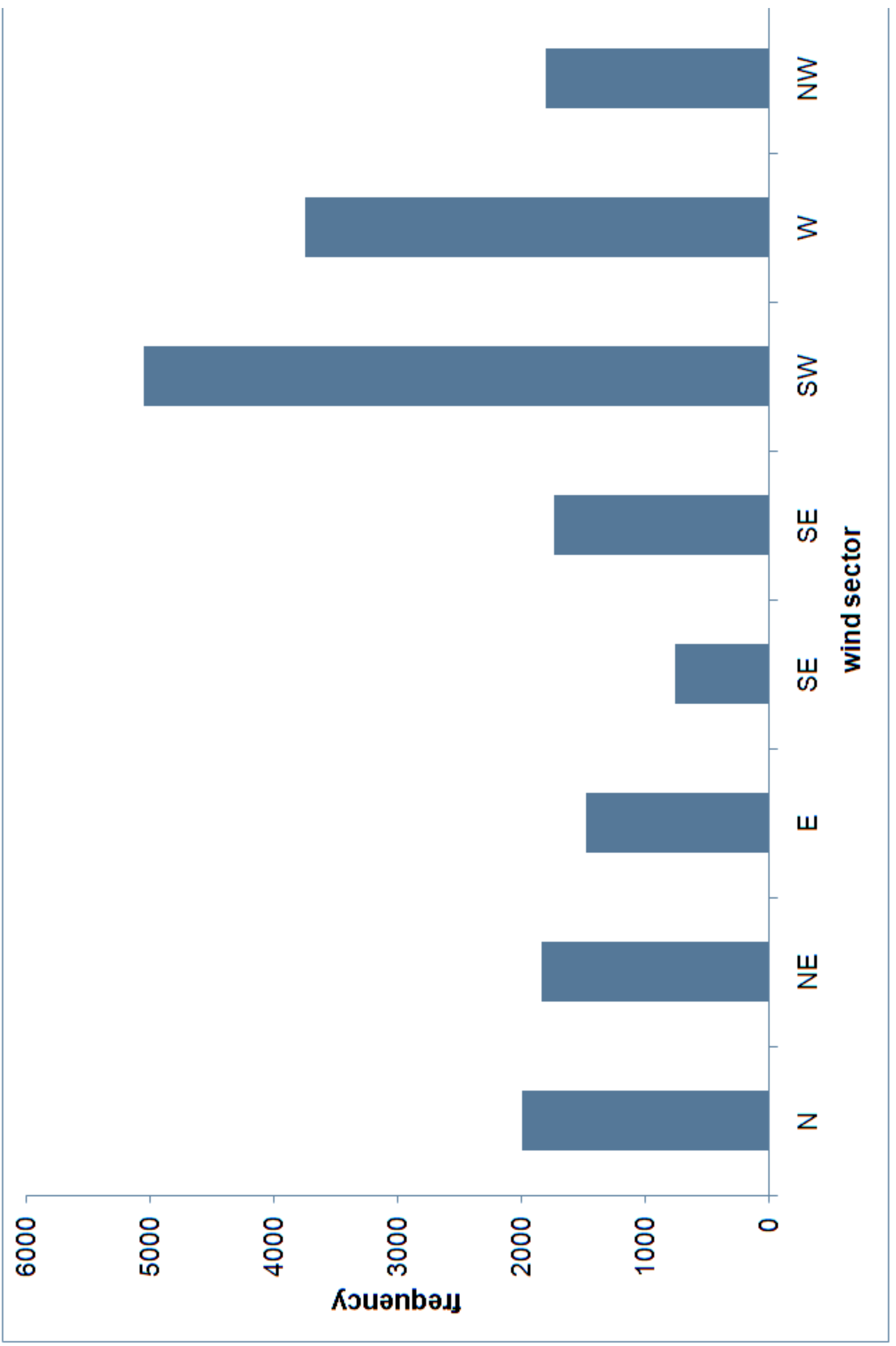




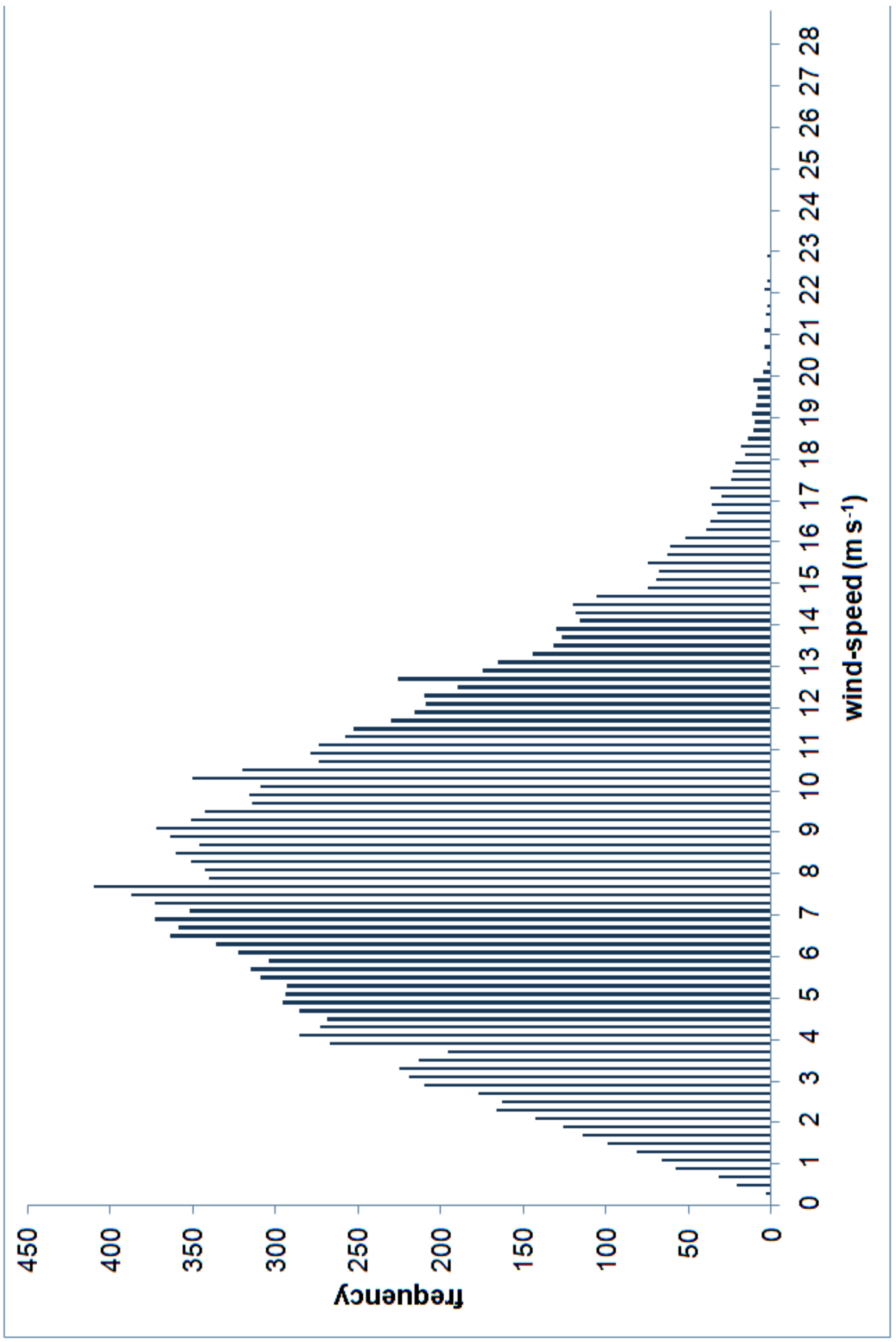




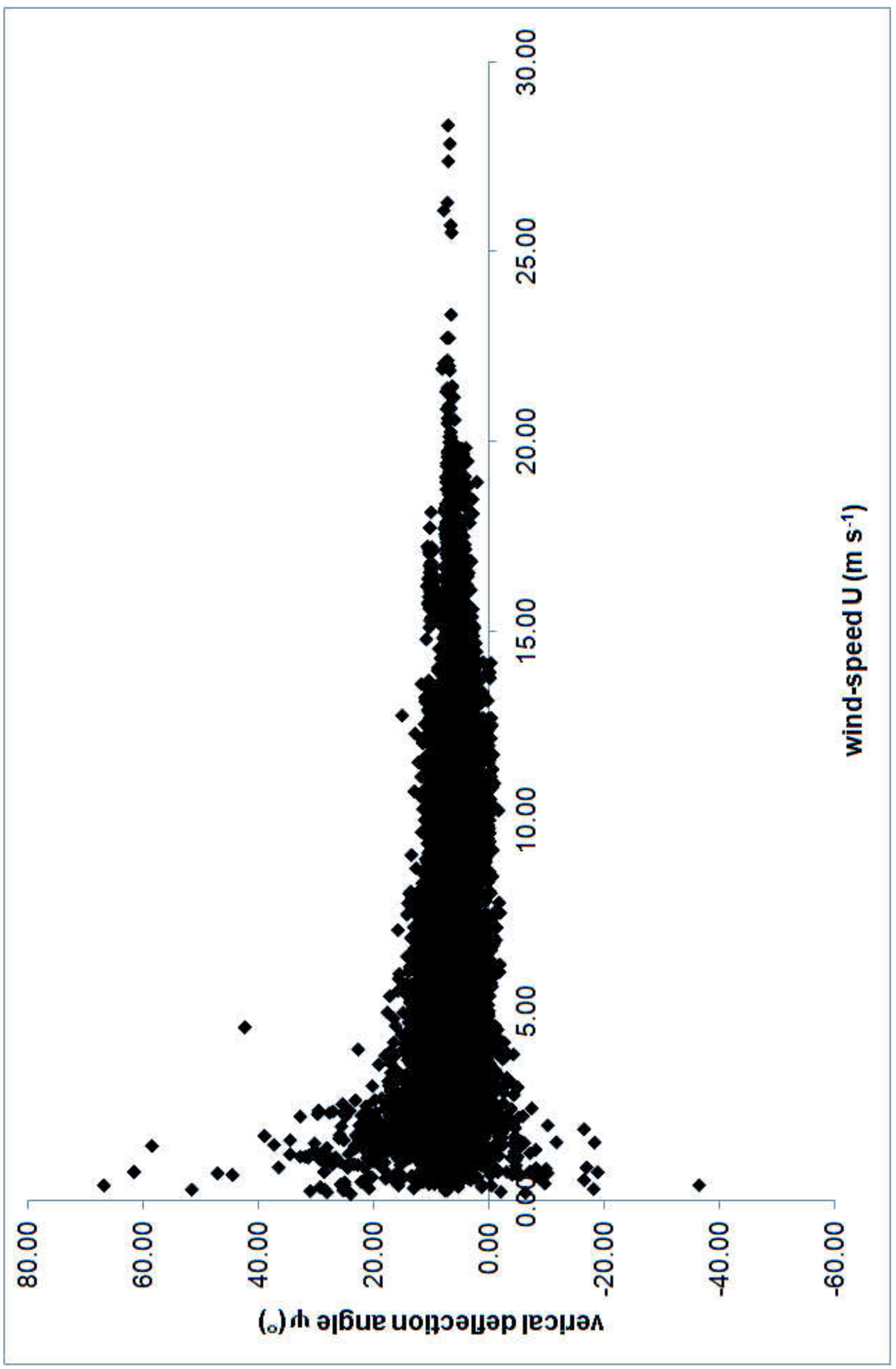




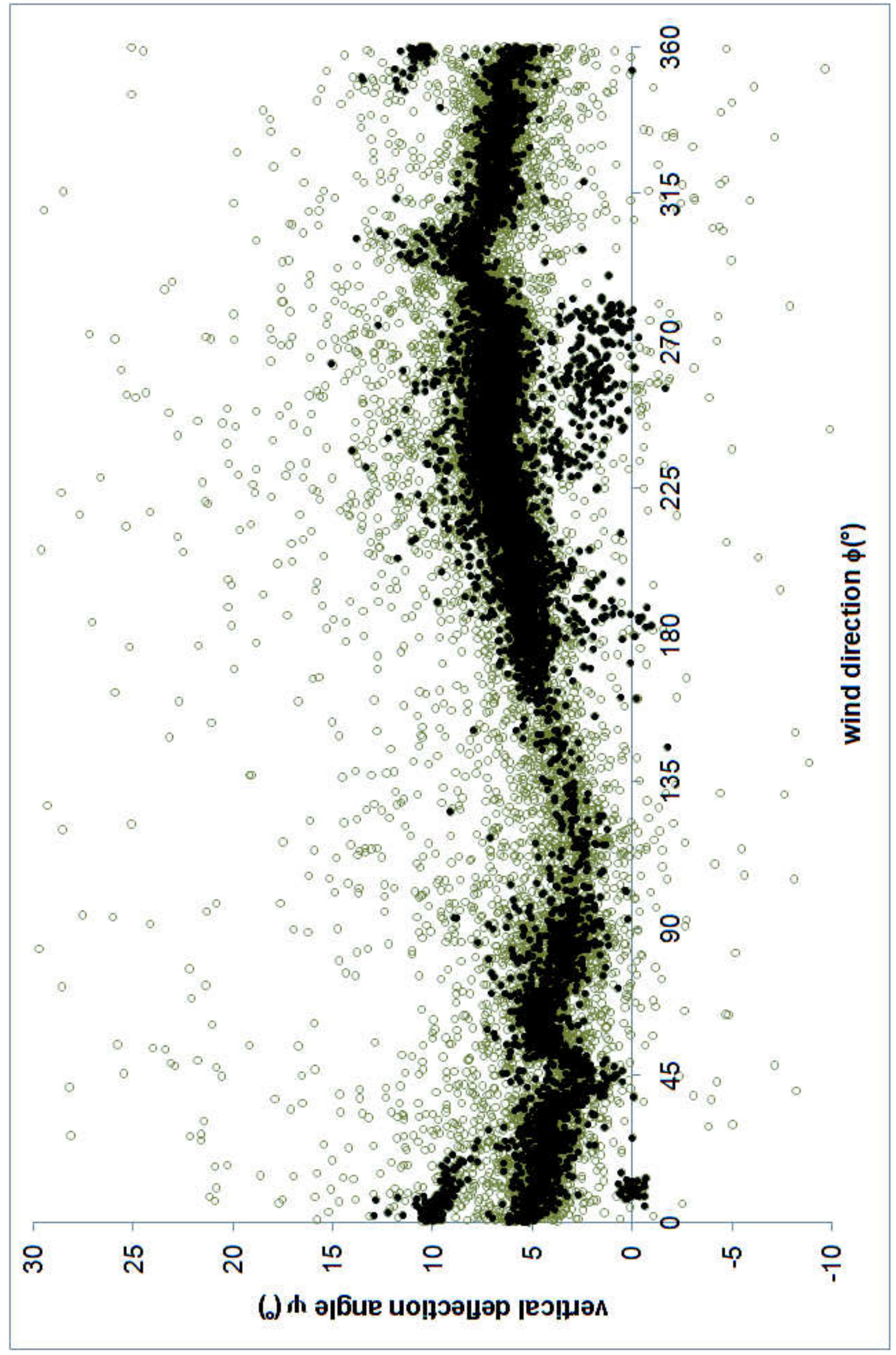




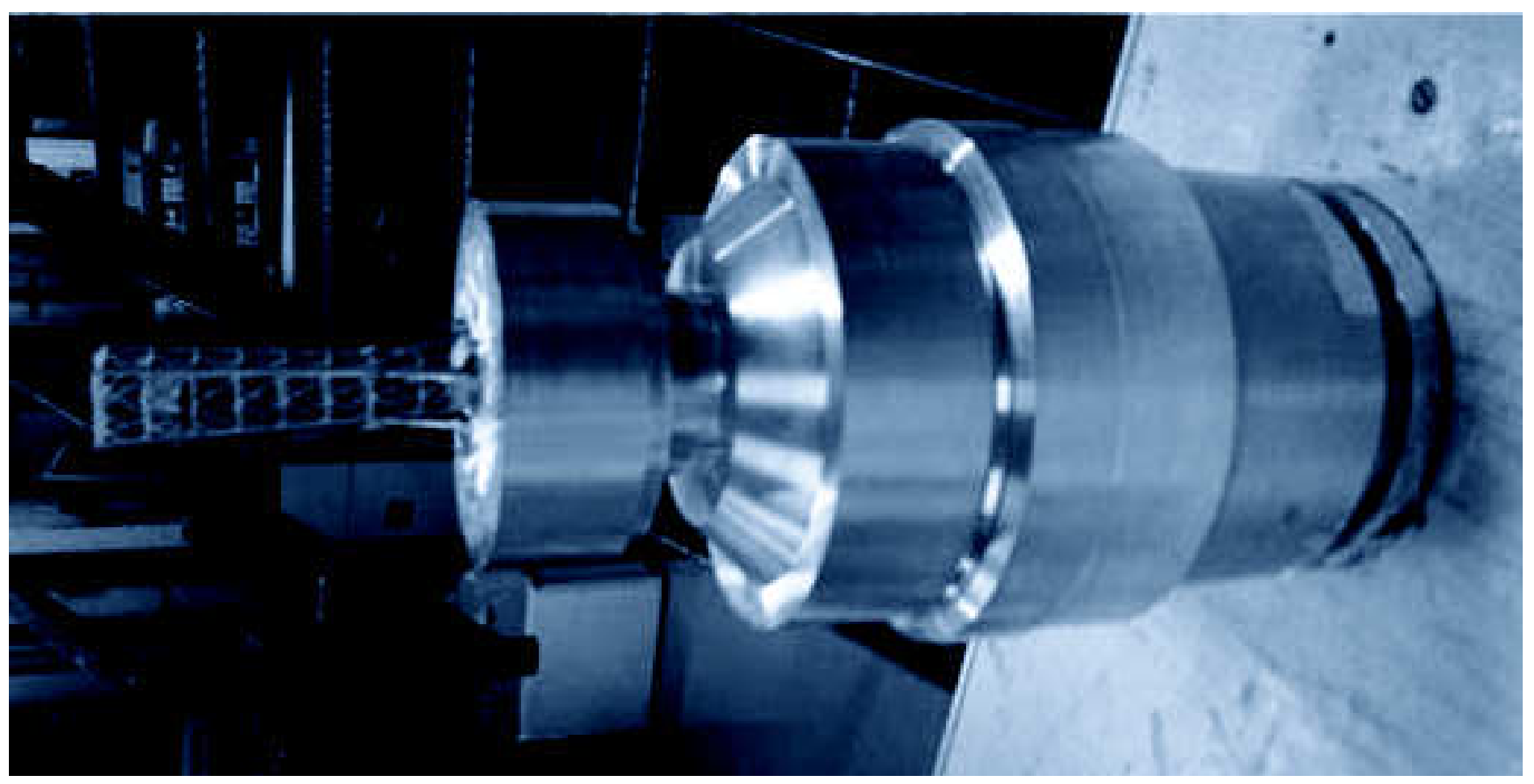




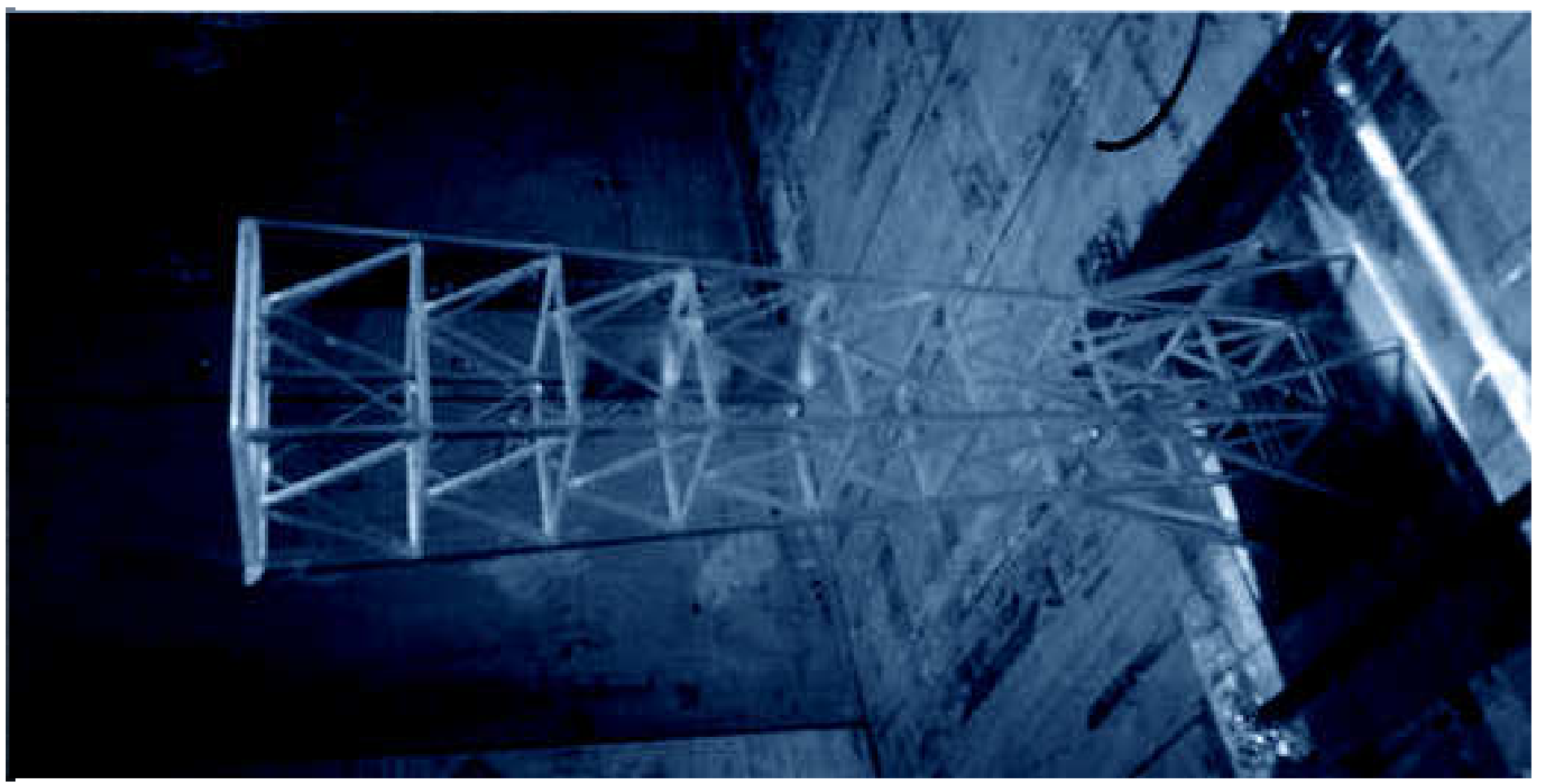




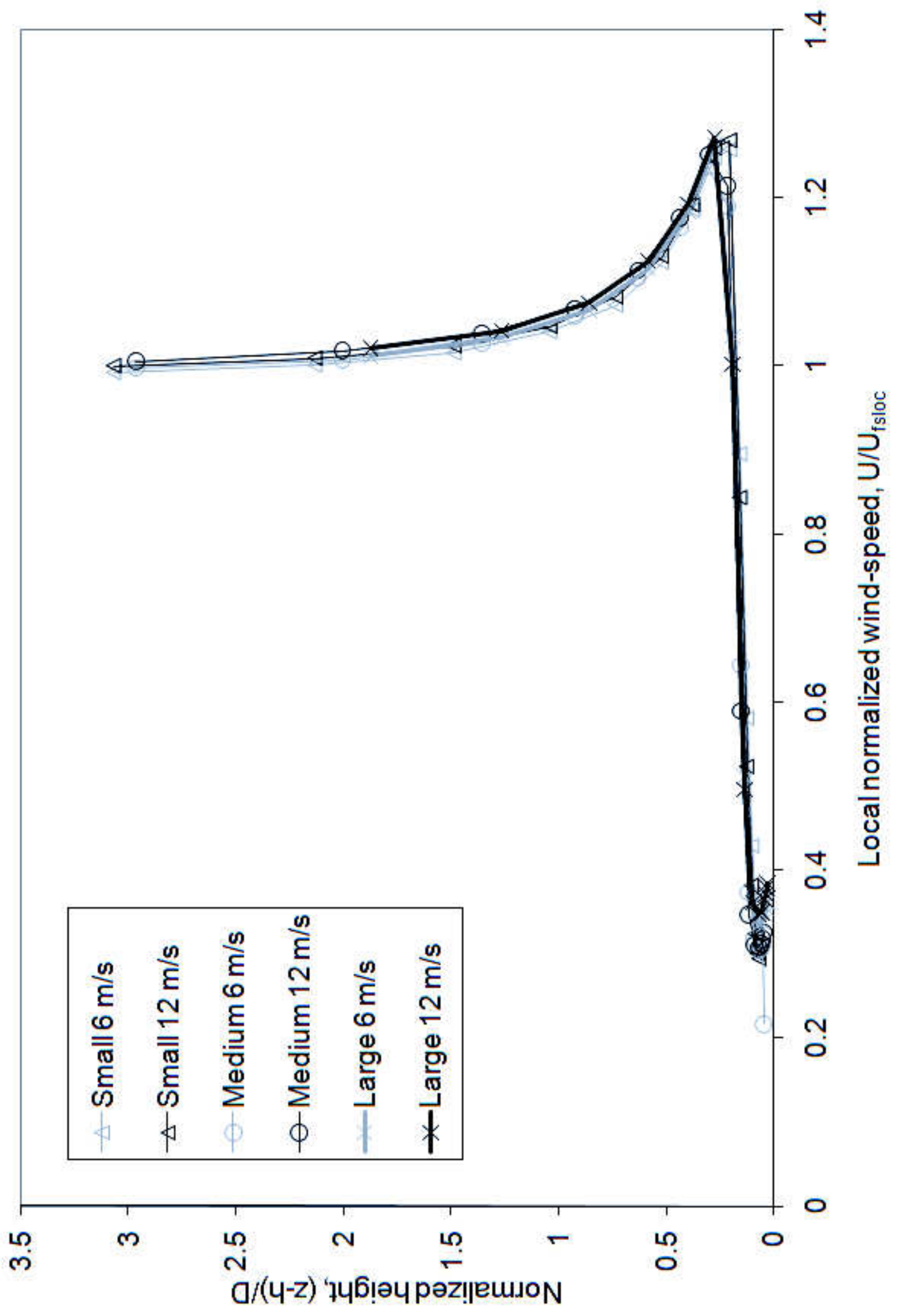




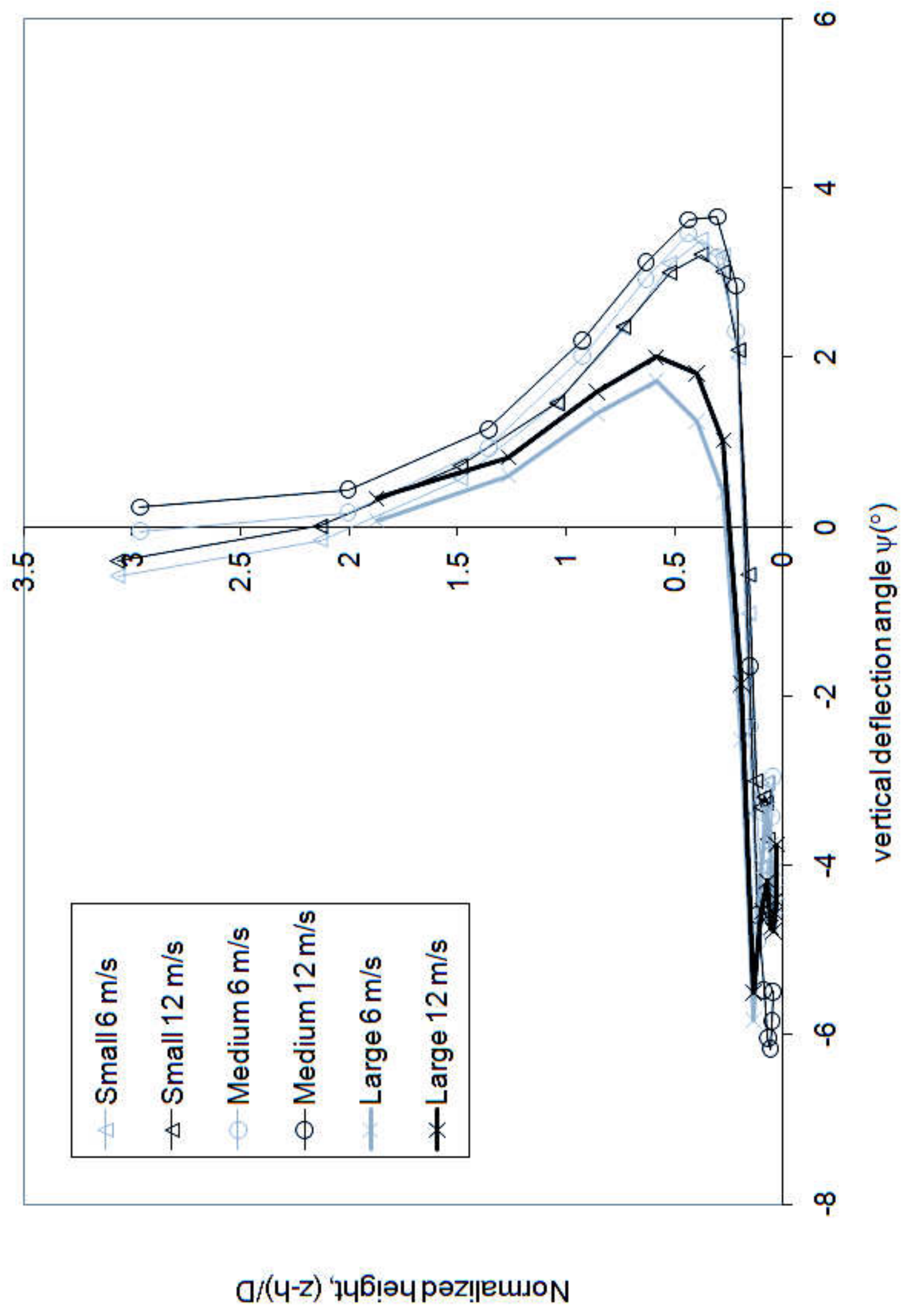




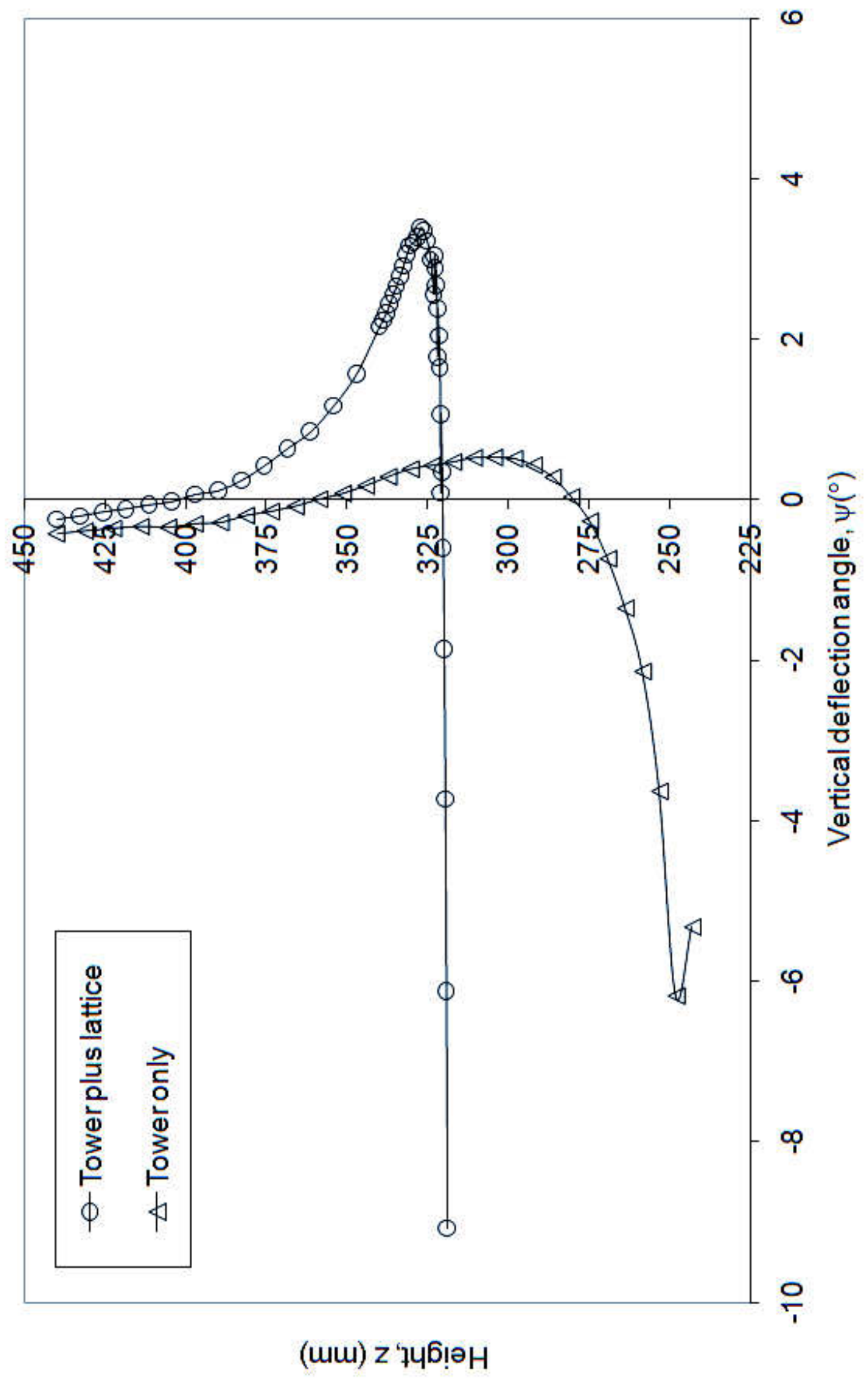




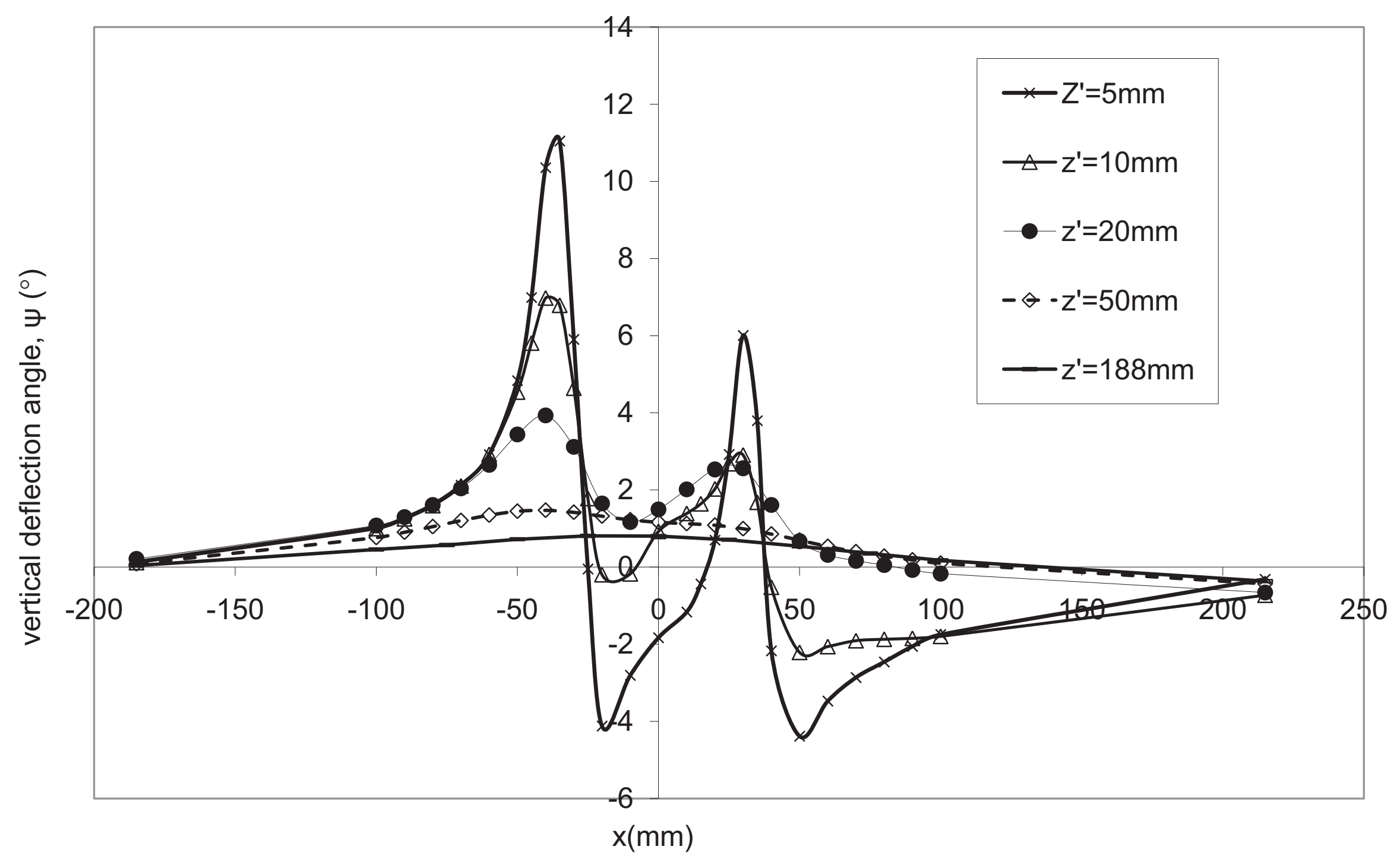




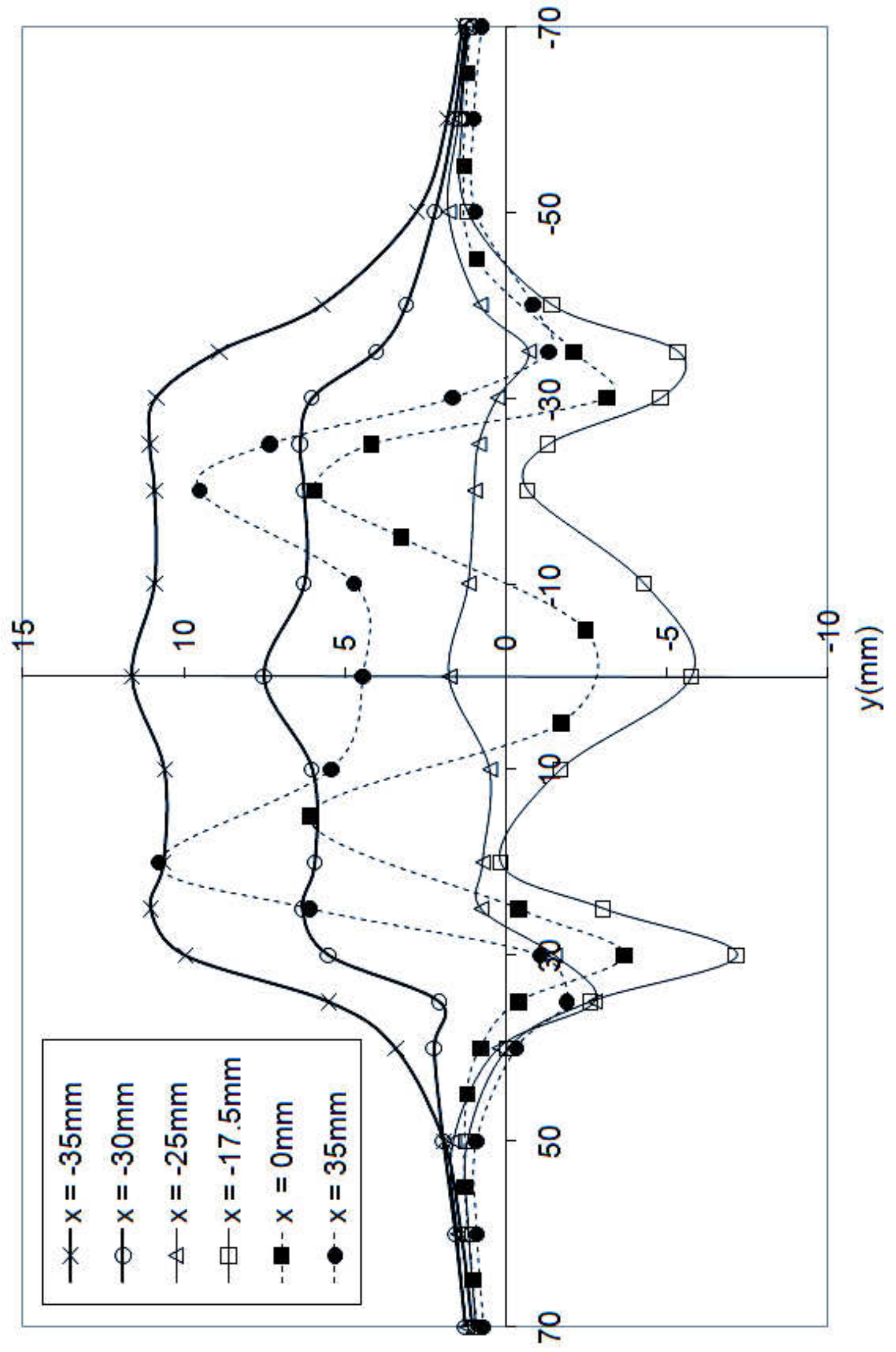

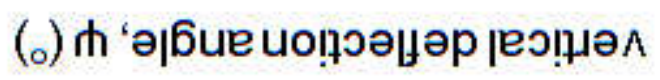




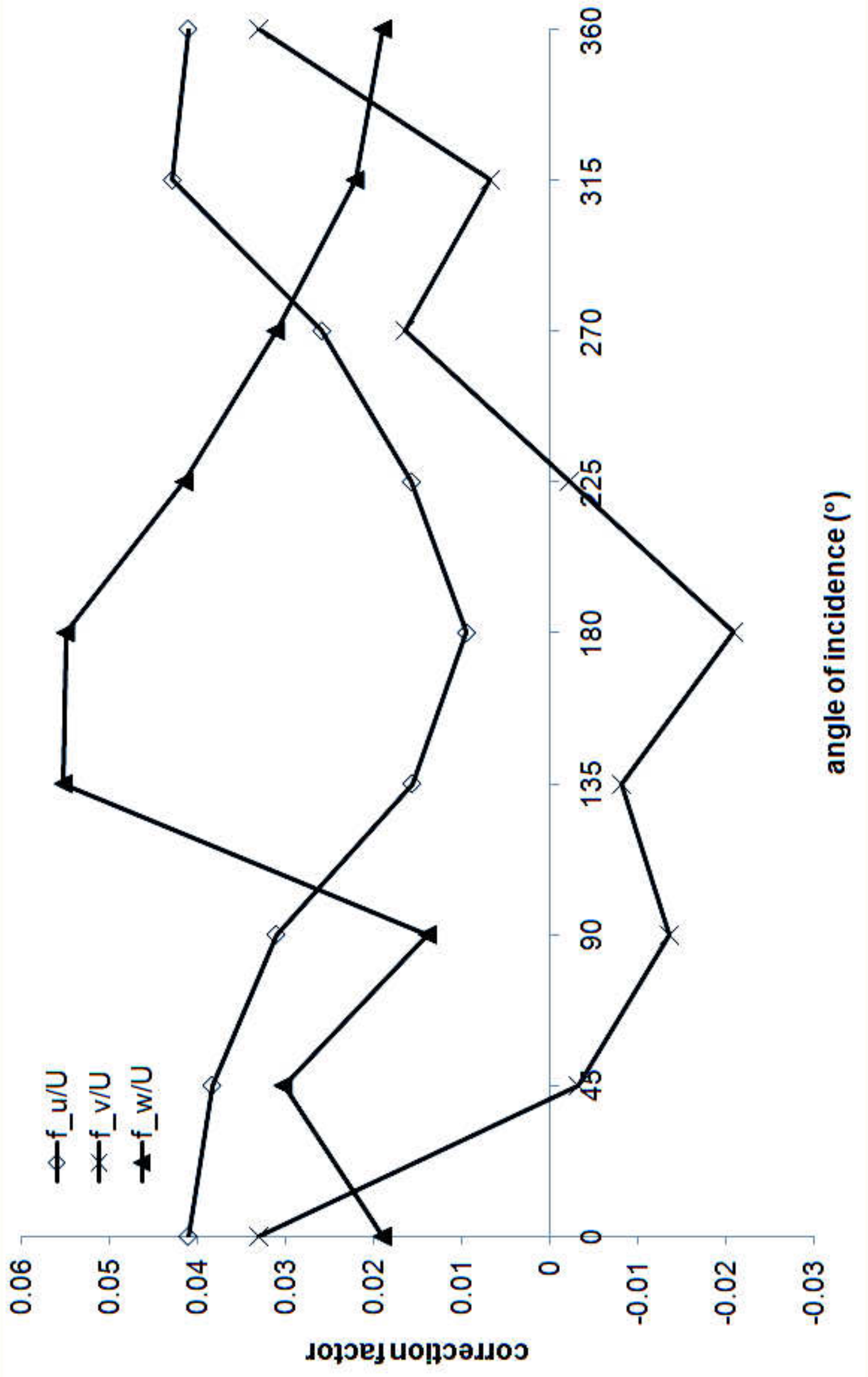




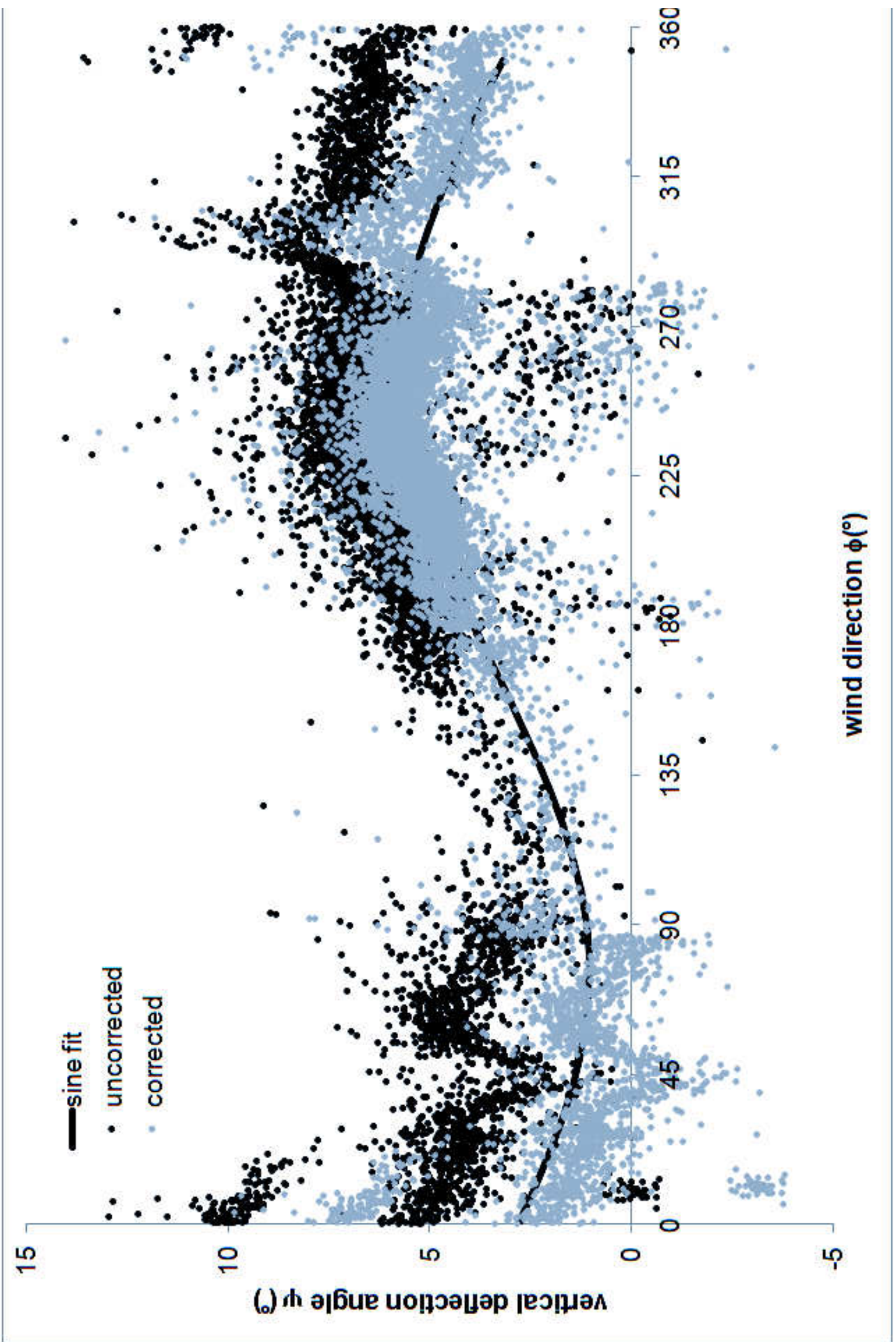


A wind-tunnel study of flow distortion at a meteorological sensor on top of the BT Tower, London, UK

The present document is the version submitted to Journal of Wind Engineering and Industrial Aerodynamics. Copyright (C) 2011 Elsevier B.V.

Final version: $\underline{\text { http://dx.doi.org/10.1016/i.jweia.2011.05.001 }}$

Barlow JF, Harrison J, Robins AG, Wood CR (2011) A wind-tunnel study of flow distortion at a meteorological sensor on top of the BT Tower, London, UK. J Wind Eng Indutr Aerodyn, 99: 899-907 